\title{
Human granulocytic Anaplasmosis: a case report in Mexico
}

\begin{abstract}
Case presentation: We report a case of a 33 month old male patient that presented with a history of rash, diarrhea, fever and lymphadenopathies, admitted to the pediatric unit for a suspected rickettsial infection. The patient was put under empirical antibiotic therapy and underwent analysis, including bonemarrow biopsy, to determine the diagnosis
\end{abstract}

Diagnosis: human granulocytic anaplasmosis

Conclusion: Tick borne diseases should be a differential diagnosis in patients presenting with rash and febrile illness with rapid clinical deterioration, especially by clinicians in the north west of Mexico and Mexico-United States border towns.

Keywords: anaplasmosis, zoonoses, tick-borne diseases
Volume 9 Issue I - 2019

\author{
Carmen Gorety Soria-Rodríguez,' Natalia \\ Galarza, ${ }^{2}$ Simi Dhillon, ${ }^{2}$ Yolanda Correa- \\ Bautista, ${ }^{3}$ Ricardo de León-Figueroa, ${ }^{4}$ María \\ Elena Haro-Acosta, ${ }^{5}$ Joan Dautt Silva, ${ }^{6}$ Luis \\ Tinoco-Gracia ${ }^{7}$ \\ 'Pediatrician and Coordinator in Health Research at IMSS \\ Hospital Gineco-Pediatria y Medicina Familiar, México \\ ${ }^{2}$ Family Medicine, Yuma Regional Medical Center, United States \\ ${ }^{3}$ Pediatric Allergist, IMSS Hospital Gineco-Pediatría y Medicina \\ Familiar, México \\ ${ }^{4}$ Pediatric Hematologist, IMSS Hospital Gineco-Pediatría y \\ Medicina Familiar, Mexico \\ ${ }^{5} \mathrm{Sc}$.D., Pediatrician and Coordinator in Health Research at IMSS \\ Delegación Baja California, México \\ ${ }^{6}$ Medical Intern, IMSS Hospital Gineco-Pediatría y Medicina \\ Familiar, México \\ ${ }^{7}$ Sc.D., Veterinarian, Veterinary Science Research Institute, \\ Universidad Autónoma de Baja California, México
}

Correspondence: María Elena Haro Acosta, Sc.D., Pediatrician and Coordinator in Health Research at IMSS Delegación Baja California, Mexicali, BC, México

Emailmaria.haroa@imss.gob, lenaharo@live.com.mx

Received: January 15, 2019 | Published: February 13,2019

\section{Introduction}

Human Granulocytic Anaplasmosis (HGA) (previously known as Human Granulocytic Ehrlichiosis, or HGE) is an infectious disease caused by Anaplasma phagocytophilum (previously Rickettsia phagocytophilum in the 1950s, then Ehrlichia phagocytophilum until 2001), a gram-negative obligate intracellular bacteria that is typically transmitted to humans by at least three kinds of ticks, including Ixodes scapularis, Ixodes pacificus, and Dermacentor variabilis, which also transmit Lyme Disease. ${ }^{1}$ Cases reported to the Centers for Disease Control and Prevention (CDC) have increased steadily since it became a notifiable disease in 2000 . The disease is curable if treatment is administered timely, and doxycycline is the number one choice of treatment even in pediatric patients. ${ }^{2}$

\section{Case description}

A 33 month old Mexican male presented to the Hospital de GinecoPediatria y Medicina Familiar No. 31, of the Mexican Institute of Social Security (IMSS) in Mexicali, Baja California, México with a week history of fever ranging from 38.5 to $39^{\circ} \mathrm{C}$ and a non-pruritic macular rash which affected palms and soles, non-bloody diarrhea, and poor appetite. The rash appeared five days after having traveled to a rural area. In the patient's medical history, it was noted that had an upper respiratory infection treated a week prior, which was treated with ampicillin and acetaminophen, as well as amoebiasis when he was 6 months old. Birth history was unremarkable and he was up-todate on immunizations. Family history was non-contributory and he had no known sick contacts. The patient lived at home with his parents and three older siblings in an urban neighborhood a block away from a junkyard and auto body shop. The family had one dog as a pet that who had recently had a litter of pups that all died within two weeks of being born. During physical examination, he had a distended and tympanic abdomen. He had a single umbilicated lesion on the ventral aspect of his right forearm. In the emergency department, he was found to have proteinuria of $100 \mathrm{mg} / \mathrm{dL}$ and leukocytosis to $11.0 \mathrm{~K} /$ $\mathrm{uL}$. The remainder laboratory studies were normal. He was diagnosed with gastroenteritis and an unspecific exanthema and treated with intravenous fluids, non-steroidal anti-inflammatory, hydrocortisone, and diphenhydramine.

\section{Clinical progression}

He was admitted in the pediatric unit for further evaluation and treatment, and started empirically on intravenous cefotaxime under the suspicion of a bacterial infection. During this period, his white blood cell count increased to $14.8 \mathrm{~K} / \mathrm{uL}$, yet he was discharged from the hospital five days later due to clinical improvement and without a specific diagnosis. He was re-admitted four days later due to a worsening general condition. His symptoms now included three emetic episodes, headaches, generalized malaise, myalgias, arthralgias, and oral intake intolerance. Physical examination noted that the child was in moderate to severe distress, an axillary temperature of $39^{\circ} \mathrm{C}$, dry oral mucosa, cervical, axillary and inguinal lymphadenopathies, hepatosplenomegaly, and a persistent generalized macular rash (Figures 1A-1C). Laboratories revealed lymphocytosis $(24.46 \mathrm{~K} /$ $\mathrm{uL})$, mild elevation of AST $(65 \mathrm{U} / \mathrm{L})$ and an elevated LDH $(413 \mathrm{U} / \mathrm{L})$. The rest of the laboratory work-up was unremarkable. At this point, the patient was suffering from systemic inflammatory response syndrome. In order to rule-out specific infectious etiologies, blood, urine, cerebrospinal fluid, pharyngeal, stool, and bronchial cultures were obtained, which turned out negative. Serologies were negative for CMV, EBV, HIV, and HSV. 

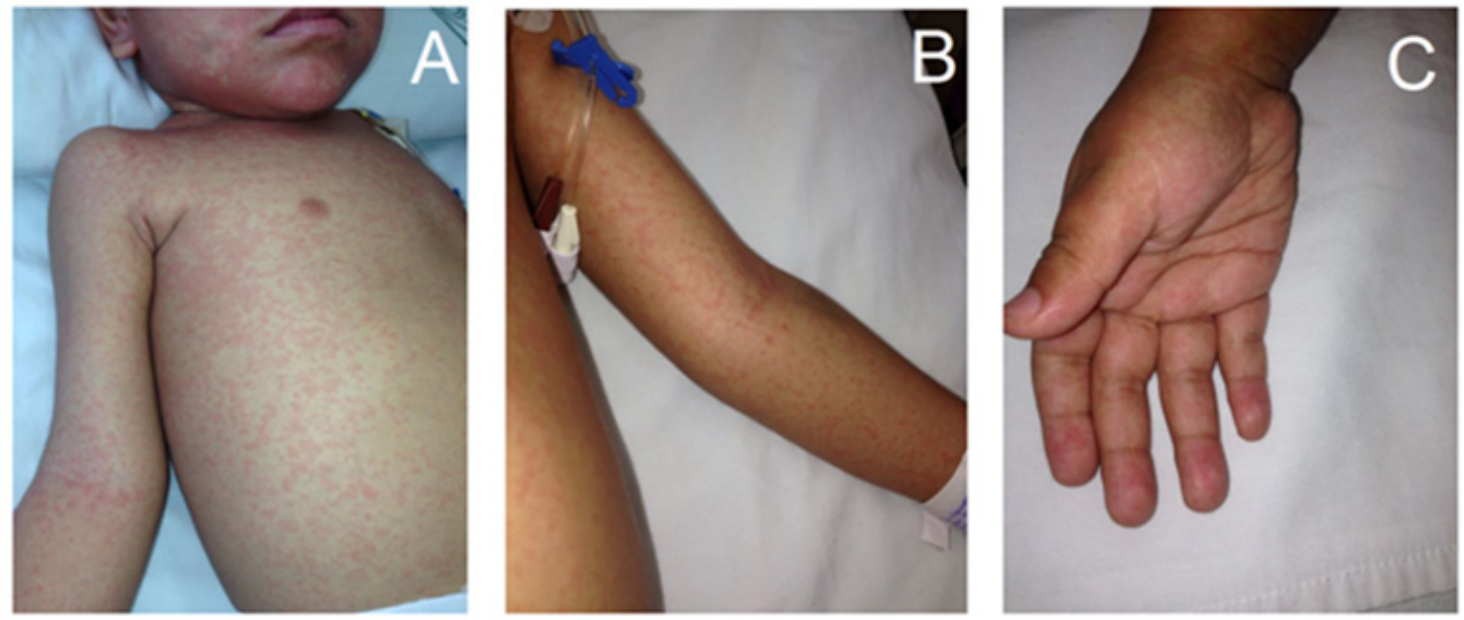

Figures IA Face and chest showing macular rash, IB Left arm with rash affecting the elbow crease, IC Palm of the right hand showing rash involvement.

Twelve days later, rickettsial polymerase chain reaction (PCR) came back negative. Two weeks later, the patient underwent a lymph node biopsy which was consistent with reactive follicular hyperplasia; a second opinion by another pathologist reported reactive lymphoid hyperplasia. A week later, a thoracoabdominal CT scan revealed multiple lymph nodes, all less than an inch in diameter, perihilar and bibasilar alveolar infiltrates with consolidation zones. In the abdomen, the liver appeared homogenous but enlarged without focal or diffuse lesions. Scattered lymph nodes were seen in the mesenteric and inframesocolic chain.

The patient underwent bone marrow aspiration given the leukocytosis and lymphadenopathies, which revealed a reactive bone marrow with predominance of neutrophils and immature forms. In addition, intracytoplasmic inclusions in granulocytes were found (Figure 2). Based on this pathology report, six days later, infectious disease specialists obtained PCR samples, which were sent out to the local school of veterinary medicine's laboratory, in search for Ehrlichia spp. and Anaplasma spp. Results were positive for Anaplasma spp. and negative for Ehrlichia spp. An additional PCR for Anaplasma phagocytophylum was obtained, which was also positive. (Figure 3 ) The patient was finally diagnosed with Human Granulocytic Anaplasmosis and treated with oral doxycycline $2.2 \mathrm{mg} /$ $\mathrm{kg}$ every 12 hours for 14 days according to CDC guidelines and made a full recovery.

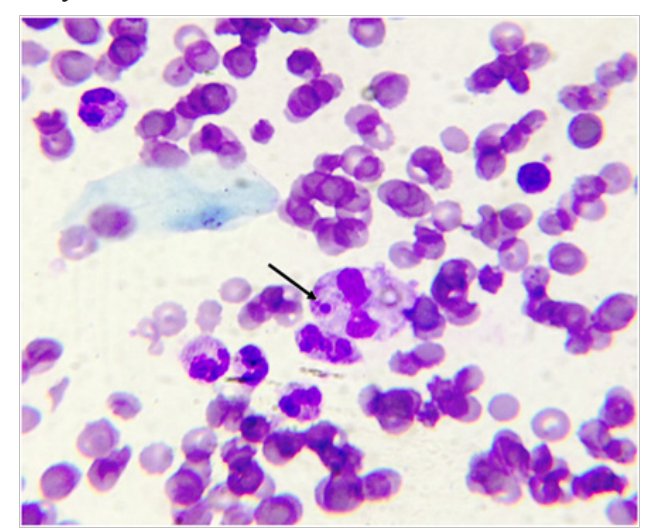

Figure 2 Bone marrow aspirate smear showed presence of intracytoplasmic granulocytic inclusions.

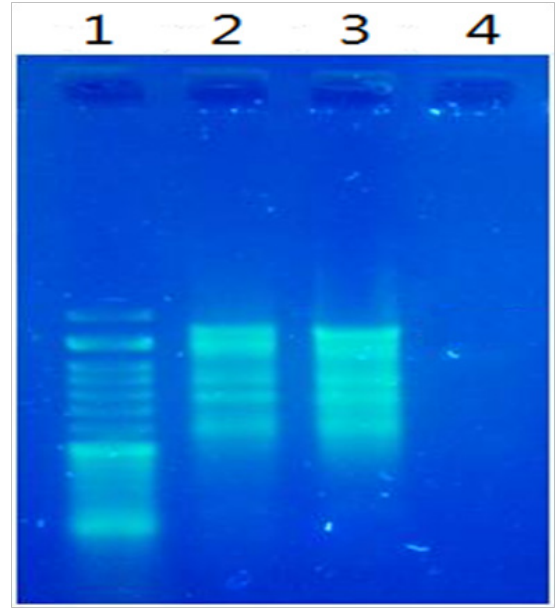

Figure 3 Positive non-nested PCR for Anaplasma phagocytophylum (I,435 base pairs). Numbers I to 4 show: marker, patient's DNA, positive control and negative control.

\section{Discussion}

Ehrlichiosis is a general term utilized for zoonotic infections caused by intracellular bacteria from the Anaplasmataceae family, including Ehrlichia chaffeensis, Ehrlichia swingier, and Anaplasma phagocytophylum, which target leukocytes. The type of affected leukocyte depends on the species. E. chaffeensis infects monocytes and is therefore named monocytic ehrlichiosis. Anaplasma phagocytophylum infects granulocytes and is therefore named granulocytic anaplasmosis or granulocytic ehrlichiosis. Both are transmitted by ticks, including Ixodes scapularis, Ixodes pacificus, and Dermacentor variabilis, which also transmit Lyme disease. ${ }^{3}$ Rickettsiosis encompasses a cluster of different diseases transmitted by vectors that can cause typhoid fever or different kinds of spotted fever (i.e. Rocky Mountain spotted fever, [RMSF]). Some characteristic symptoms or history elements are high-risk behavior, fever, headache, myalgias, arthralgias, rash, multiorgan involvement in late phases of diseases, usually after the second week. ${ }^{4}$

In this patient, we asked for rickettsia PCR that was collected 48 hours after initiating antibiotic treatment with cefotaxime, a 
third generation cephalosporin with documented activity against Rickettsia and an accepted second line medication for RMSF. IgG and IgM serology for Rickettsia rickets was not obtained. Historically, anaplasmosis has not been related with dermatological manifestations, usually if they are dermatological symptoms involved, it means there is a coexisting condition with RMSF. It should be noted that, during the time this case developed, there was a unique outbreak of RMSF in Mexicali. ${ }^{5}$ The progression of disease, 22 days in total, included persistent fever, worsening of general condition, respiratory distress, increasing hepatosplenomegaly and lymphadenopathies, and worsening of laboratory values. This caused the frustration to determine etiology to rise, especially due to the recent local outbreak of RMSF at the time. The differential diagnosis for Rickettsiosis is broad based on the unspecific signs and symptoms. For example, infection with S. aureus can lead to toxic shock syndrome, which is manifested as fever, hypotension, renal insufficiency, and multi organ failure. It is often accompanied by aqueous diarrhea and generalized rash. Similarly, Kawasaki disease, a systemic vasculitis that mainly affects mediumsized arteries, can also lead to multi organ complications. Criteria for diagnosis includes fever $>5$ days without evident cause, polymorphous rash, cervical lymphadenopathy, conjunctivitis without discharge, edema and erythematic of extremities, and ultimately, periungual desquamation. Oropharyngeal manifestations include swollen lips, pharyngeal hyperemia, and strawberry tongue. Laboratory studies in Kawasaki Disease often reveal elevated WBC with increased immature forms, increased erythrocyte sedimentation rate and protein $\mathrm{C}$, normal platelets in the first week and thrombocytosis as the disease progresses, as well as elevation of LDH. Infectious mononucleosis, which results from infection with cytomegalovirus, Epstein-Barr virus, and others, is characterized by fever, pharyngitis, multiple lymph nodes, splenomegaly, rash, leukocytosis with increased monocytes and atypical lymphocytes. ${ }^{6}$ The treatment for both Ehrlichiosis and Rickettsiosis is doxycycline, tetracycline, or rifampin (for patients with contraindication to doxycycline or tetracycline like allergies or pregnancy). ${ }^{7}$ It consists of a 5 to 14 day course of doxycycline $100 \mathrm{mg}$ IV or PO every 12 hours for adults and $2.2 \mathrm{mg} / \mathrm{kg}$ PO every 12 hours in the pediatric population, tetracycline $500 \mathrm{mg}$ PO every 6hours for adults and $25-50 \mathrm{mg} / \mathrm{kg} /$ day PO divided into 4 doses in the pediatric population for 5-14days, and rifampicin 300mg PO every 12 hours in adults and $10 \mathrm{mg} / \mathrm{kg}$ PO every 12 hours for 7-10days in the pediatric population. ${ }^{3}$ An answer that the emergency department in this hospital implemented for this type of cases (high clinical suspicion, but negative serology or other diagnostic studies) was to introduce an algorithm for the timely diagnosis of rickettsia infections based on a high clinical suspicion since the disease is now endemic to the area.

\section{Conclusion}

Tick borne diseases have not traditionally been suspected in the Southwestern United States, and have only recently been made a differential diagnosis that has to be considered by clinicians in the north west of Mexico. Because of the unique border dynamics that occur in this region, increased awareness of the disease by health care providers on either side of the international border is needed in order to identify those symptomatic patients, as well as those at risk of developing the disease.

\section{Acknowledgments}

None.

\section{Conflicts of interest}

We have no conflicts of interest to disclose.

\section{References}

1. Wormser GP, Dattwyler RJ, Shapiro ED, et al. The clinical assessment, treatment, and prevention of lyme disease, human granulocytic anaplasmosis, and babesiosis: clinical practice guidelines by the Infectious Diseases Society of America. Clin Infect Dis. 2006;43(9):1089-1134.

2. Centers for Disease Control and Prevention. Anaplasmosis. 2019.

3. Centers for Disease Control and Prevention. Tickborne Diseases of the US: A Reference Manual for Health Care Providers. 2019.

4. Davis BP, Marx JA. An Evidence Based Approach to the diagnosis and treatment of Rocky Mountain Spotted Fever In the Emergency Department. Emerg Med Pract. 2007;9(3):1-24.

5. Faccini ÁA, García L, Hidalgo M, et al. Syndromic classification of rickettsioses: an approach for clinical practice. Int $J$ Infect Dis. 2014;28:126-139

6. Eremeeva ME, Zambrano ML, Anaya L, et al. Rickettsia rickettsia in Rhipicephalus Ticks, Mexicali, Mexico. $J$ Med Entomol. 2011;48(2):418-421.

7. Centers for Disease Control and Prevention. New Research on Doxycycline. 2019. 